\title{
Cx43 expressed on bone marrow stromal cells plays an essential role in multiple myeloma cell survival and drug resistance
}

\author{
Jinxiang $\mathrm{Fu}$
}

Department of Hematology, No. 2 Affiliated Hospital of Soochow University, Suzhou, China

Submitted: 18 December 2014

Accepted: 25 January 2015

Arch Med Sci 2017; 13, 1: 236-245

DOI: 10.5114/aoms.2017.64722

Copyright ๑ 2016 Termedia \& Banach

\author{
Corresponding author: \\ Prof. Jinxiang Fu \\ Department of Hematology \\ No. 2 Affiliated Hospital \\ of Soochow University \\ 1055\# Sanxiang Rd \\ 215004 Suzhou, China \\ Phone: +8651267784627 \\ E-mail: fjx3000@126.com
}

\begin{abstract}
Introduction: Connexin-43 (Cx43), a connexin constituent of gap junctions (GJs) is mainly expressed in bone marrow stromal cells (BMSCs) and played a important role on hematopoiesis. In this study, we explored the role of gap junctions (GJs) formed by CX43 between BMSCs and multiple myeloma (MM) cells.

Material and methods: $\mathrm{qPCR}$ and western blot assays were employed to assay Cx43 expression in three MM cell lines (RPMI 8266, U266, and XG7), freshly isolated MM cells, and bone marrow stromal cells (BMSCs). Cx43 mRNA and proteins were detected in all three MM cell lines and six out of seven freshly isolated MM cells.

Resuths: The BMSCs from MM patients expressed $\mathrm{C} \times 43$ at higher levels than of normal donor (ND-BMSCs). Dye transfer assays demonstrated that gap junction intercellular communication (GJIC) occurring via $\mathrm{C} \times 43$ situated between MM and BMSCs is functional. Cytometry beads array (CBA) assays showed that cytokines production changed when the ND-BMSCs were co-cultured with MM cells, especially the levels of IL-6, SDF-1 $\alpha$ and IL-10 were higher than those the cells cultured alone and decreased significantly in the presence of GJ inhibitor heptanol. Our results demonstrated that the cytotoxicity of BTZ to MM cells decreased significantly in the presence of BMSCs, an effect that was partially recovered in the presence of GJ inhibitor. Conclusions: Our data suggest that GIIC between MM and BMSCs is a critical factor in tumor cell proliferation and drug sensitivity, and is implicated in MM pathogenesis.
\end{abstract}

Key words: connexins, gap junctions, mesenchymal stromal cells, plasma cell neoplasms, microenvironment.

\section{Introduction}

Multiple myeloma ( $M M)$, a fatal hematological malignancy that develops within the bone marrow (BM) microenvironment, is characterized by the uncontrolled clonal proliferation of malignant plasma cells within the BM. Despite the presence of a variety of chromosomal aberrations, translocations, and mutations in essential growth and tumor suppressor genes in MM cells, oncogenomic studies have identified few differences in distinguishing monoclonal gammopathy of unknown significance from that of MM [1-3]. This finding highlights the essential role of the BM mi- 
croenvironment in disease maintenance and progression. Indeed, direct and indirect interactions among MM cells and other cells within the liquid milieu of the BM environment are key requirements for MM pathogenesis, cell growth, survival, migration, and drug resistance. The extent to which this microenvironment becomes supportive of MM growth, as well as the contribution and interaction of the variety of components within the BM microenvironment to enhancing $M M$ growth, are only now beginning to be understood [4-6]. The BM microenvironment is composed of generically denominated bone marrow stromal cells (BMSCs). Cell-to-cell contact between BMSCs and MM cells has been recently proposed as a pivotal regulatory interaction in the growth and survival of malignant plasma cells.

Gap junctions (GJs) are membrane-spanning channels that facilitate intercellular communication by establishing continuity of cytoplasms between communicating cells and allowing small signaling molecules to pass from cell to cell, representing the best-known intercellular communication system [7]. Over the past two decades, many studies have described a role for $G$ J intercellular communication (GIC) in the proliferation and differentiation of a variety of cells, including bone cells. Cancelas et al. [8-12] have characterized the expression of 11 different connexins (CXs) in different stromal cells derived from murine bone marrow and fetal liver, but only three $\mathrm{Cxs}$ were detected in the bone marrow cells: Cx31, Cx43, and Cx45. Cx43 in particular was reported to have a supportive function in normal hematopoiesis, so GJs thereby contribute to the stromal regulation of clonal growth of hematopoietic progenitors. Ciovacco et al. [13] demonstrated the functionality of GIIC between megakaryocytes (MKs) and osteoblasts (OBs), and that inhibition of GIC in MK/OB cultures enhanced OB proliferation. Recently, Hecht et al. [14] showed that interaction with OBs enhances the capability of myeloma cells to transmigrate and invade across type I collagen. Our previous studies [15, 16] also demonstrated that OBs induced from BM mesenchymal stem cells supported migration and proliferation of MM cells; in particular, the alteration of $\mathrm{C} \times 43$ expression in BMSCs is involved in the interaction of MM cells with the BM environment. However, the specific role of Cx43 in the growth and survival of MM cells remains largely unknown.

Therefore, we investigated whether MM cells are capable of communicating with BMSCs through GJs, and whether MM-mediated GIIC was responsible for BMSC-induced enhancement of MM proliferation and drug resistance. Here, we demonstrate that MM cells express $C \times 43$ and communicate with BMSCs through GJs, and we explored the mechanisms by which MM cells interact with BMSCs.

\section{Material and methods}

\section{Preparation of bone marrow stromal cells}

Bone marrow aspirates were obtained from $7 \mathrm{pa}$ tients ( 4 male and 3 female patients, average age: 57 years) with $M M$ at diagnosis and 5 donors from patients with rib resection or amputation without hematological disease ( 2 male and 3 female, mean age 50 years) after formal written consent was obtained, in accordance with the guidelines of the Ethics Committee of Soochow University. Mononuclear cells (MNCs) were separated by Ficoll-Hypaque density gradient centrifugation. To extract hematopoietic stem cells and prevent overgrowth of the cultures with macrophages, CD45+ cells were depleted by negative immunomagnetic cell selection using the Mini MACS device (Miltenyi Biotec, Bergisch Gladbach, Germany) according to the manufacturer's instructions. These cells were found to be >95\% BMSCs by a variety of criteria [17-19]. Bone marrow stromal cells were detached using trypsin (Invitrogen, Cergy-Pontoise, France), and counted using trypan blue exclusion. They were cultured once under the same conditions (first passage, P1). BMSCs at P0 or P1 were used immediately or frozen until use.

\section{Cell lines and primary specimens}

The myeloma cell lines RPMI 8226 and U266 cells were obtained from the American Type Culture Collection (Rockville, MD, USA) and cultured in RPMI-1640 medium supplemented with $10 \%$ FCS and $1 \%$ glutamine. The interleukin-6-dependent human myeloma cell line XG-7 was a gift kindly provided by Prof. Zhang of Soochow University [20]. Primary human MM cells were obtained from patients with MM. Additionally, seven heparinized BM aspirates were collected from patients with MM, and the MNCs were separated by Ficoll-Hypaque density gradient centrifugation. Primary malignant cells were isolated from MNCs by positive selection for CD138 as previously described [16]. The isolated cells were analyzed by flow cytometry (FCM) to examine the proportion of $\mathrm{CD}_{138^{+}} \mathrm{MM}$ cells (> 90\% pure; Clone: DL-101, BD Bioscience, San Jose, CA, USA).

\section{Real-time PCR and western blotting}

Connexin 43 mRNA expression in MM cell lines, freshly isolated MM cells and BMSCs was determined using standard real-time polymerase chain reaction (PCR) techniques as we previously described [16]. The primer sequences for $\mathrm{C} \times 43$ used were as follows: forward, $5^{\prime}$-CCTTTGACTTCAGCCTCCAA-3', reverse, 5'-CATGTCTGGGCACCTCTCTT-3'; GAPDH primers, forward, 5'-CGTGGGGCTGCCCAGAACAT-3', reverse, 5'-TCTCCAGGCGGCACGTCAGA-3'. 
Multiple myeloma cell lines, primary MM cells, BMSCs from normal donors (ND-BMSCs) and MM patients (MM-BMSCs) were collected according to the methods mentioned above. The western blotting assay was performed as we previously described elsewhere [16]. Antigen-antibody complexes were detected using secondary antibodies conjugated to HRP (Invitrogen) and visualized by enhanced chemiluminescence (GE Healthcare).

\section{Assays of GJIC}

We adapted our protocol from Ziambaras et al. [21]. In brief, the freshly isolated myeloma cells or cell lines were simultaneously labeled with $10 \mu \mathrm{M}$ calcein-AM (Invitrogen) and $10 \mu M$ 1,1'-dioctadecyl-3,3,3'3-tetramethylindo-carbocyanine perchlorate (Dil; Invitrogen, USA) for $25 \mathrm{~min}$ at $37^{\circ} \mathrm{C}$ in a $5 \% \mathrm{CO}_{2}$ fully humidified atmosphere. Cells were washed twice in phosphate buffered saline (PBS) prior to administration on receiving cells (BMSCs, $>90-95 \%$ confluent). A total of $2 \times 10^{4}$ of resulting cells were seeded onto the pre-cultured BMSCs and incubated for $75 \mathrm{~min}$ at $37^{\circ} \mathrm{C}$. To block GJIC, heptanol was dissolved in ethanol as described previously $[22,23]$, so that the final concentration in the cell culture medium was $0.15 \%$ ethanol with $0,5,15,25,50$, and $100 \mathrm{mM} / \mathrm{l}$ heptanol, respectively. Prior to conducting FCM studies, the cultures were examined microscopically to confirm dye transfer from myeloma cells to BMSCs. The double labeling of myeloma cells with Dil is a secondary confirmation that the donor cells are viable and that the dye is not permeating from the cells. We next proceeded to conduct FCM analysis, where the cells were trypsinized to release BMSCs, fixed in $4 \%$ paraformaldehyde, resuspended in FCM buffer, and analyzed on a FACstar Plus Flow Cytometer (Becton Dickinson, Franklin Lakes, NJ, USA). In studies utilizing heptanol as a GJ inhibitor, we found that both 50 and $100 \mathrm{mM}$ of heptanol resulted in a significant reduction in GJIC, so $50 \mathrm{mM}$ was used for inhibition in further experiments.

\section{Cytokine production}

The supernatants from ND-BMSCs or RPMI 8266 cultured alone and ND-BMSCs cocultured with RPMI 8226 cells with or without heptanol $(50 \mathrm{mM} / \mathrm{l})$ were collected. The interleukin (IL)-6, IL-10, transforming growth factor (TGF)- $\beta$, basic fibroblast growth factor (bFGF) and IL-17 concentrations were measured by Cytometric Bead Array (CBA) assay, using kits obtained from BD Biosciences Pharmingen (USA) according to the manufacturer's recommendations. The level of stromal cell-derived factor- $1 \alpha$ (SDF-1 $\alpha$ ) was determined by the ELISA assay according to the manufacturer's instructions.

\section{Colony assays}

A series of co-culture colony assays were performed with different MM cells. The ND or MMBMSCs at passage 2 were sub-cultured at 40-60\% confluence in a 24-well plate overnight. Next, 5000 RPMI 8226 and freshly isolated MM cells were seeded directly into 24-well plates and overlaid with $1 \%$ methylcellulose gel with $10 \%$ FBS in the presence or absence of $50 \mathrm{mM} / \mathrm{ml}$ heptanol. The number of colonies in each well was counted after 2 weeks under microscopic examination, with a colony defined as a cluster of at least 50 cells.

\section{Drug sensitivity assays}

We evaluated the role of GIC in bortezomib-induced MM cell apoptosis. BMSCs were seeded onto 6-well plates at a concentration of $1 \times 10^{5} /$ well. After 4-h incubation, RPMI 8266 was plated into the culture system at a dose of $1 \times 10^{5} / \mathrm{ml}$. The cohorts examined were 1) MM alone (control); 2) RPMI 8266 + BTZ; 3) RPMI $8226+$ MM-BMSCs + BTZ; 4) RPMI 8226 + MM-BMSCs + heptanol; 5) RPMI 8226 + ND-BMSCs + BTZ; 6) RPMI 8226 + ND-BMSCs + heptanol. Cells were plated in triplicate and the cultures were incubated for an additional $24 \mathrm{~h}$. Cell number and annexin V/PI binding were analyzed on FCM. The concentrations of BTZ and heptanol used in this study were $20 \mathrm{nmol} / \mathrm{l}$ and $50 \mathrm{mM} / \mathrm{ml}$ respectively.

\section{Ethical approval}

Our study programs were approved by the Ethics Committee of Soochow University Hospital. According to Soochow University Hospital committee guidelines, formal written consent was obtained from all participants prior to performing the studies.

\section{Statistical analysis}

An unpaired two-tailed Student's $t$ test was used to determine the statistical significance of differences between means. Differences were evaluated by using SPSS software version 19.0 (SPSS, Inc., USA), with a significance cutoff of $p<0.05$.

\section{Results}

\section{Expression of $\mathrm{Cx} 43$ in MM cell lines, MM cells, and BMSCs}

Cx43 expression was heterogeneous and aberrant in MM cells - specifically, in RPMI 8266, U266, and $X G 7$ cells. Six out of seven freshly isolated MM cells expressed Cx43. Cx43 expression was also observed in BMSCs, and Cx43 expression in BMSCs was higher than that observed in MM cells. 
Interestingly, BMSCs isolated from patients with MM had high expression of Cx43 compared with BMSCs from healthy controls (Figure $1 \mathrm{~A}$ ).

Western blot analysis revealed that RPMI8226, U266 and XG7 cells expressed CX43 at moderate levels. All seven primary MM cells from MM patients expressed Cx43 (Figure 1 B). Cx43 was expressed both in BMSCs from normal donors and MM patients (Figure $1 \mathrm{C}$ ). Generally, Cx43 expression in MM-BMSCs was stronger than that in ND-BMSCs $(p<0.05)$.

\section{Myeloma cells form functional GJ which can be used to communicate with BMSCs}

Visual inspection confirmed the viability of both donor and receptor cells and demonstrated the specificity of the dye transfer (Figure 2-1). Flow cytometry analysis was used to show the transfer of calcein AM from MM cells to BMSCS (Figure 2-2), demonstrating that GIIC occurs between the cells. Similar data were obtained upon replacing $M M$ cell lines with freshly isolated $M M$ cells (data not shown). In the meantime, our result confirmed the specificity of GIIC between MM

A

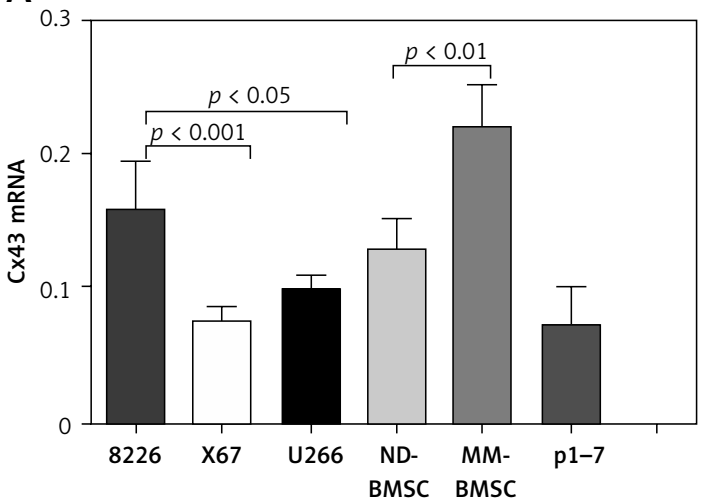

C
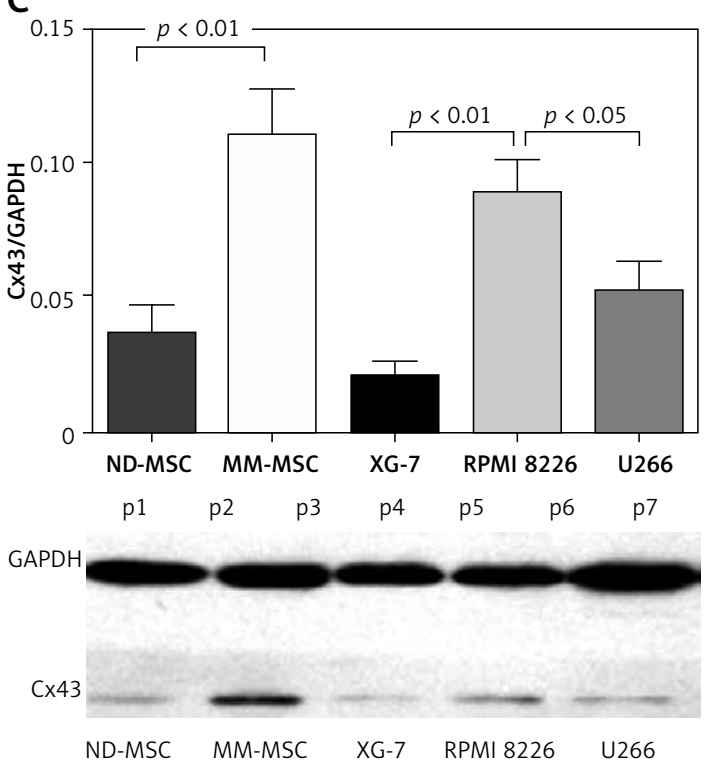

cells and BMSCs and demonstrated that inhibition of dye transfer from MM to BMSCs occurred in a dose-dependent manner. As shown in Figure 2-2, GIC remained unchanged in the range of $0-5 \mathrm{mM}$ heptanol, whereas GIIC was reduced gradually in the range of 10-25 $\mathrm{mM}$ of heptanol, until significant inhibition of GIC was observed at 50 and $100 \mathrm{mM}$ of heptanol. These experiments were also performed with freshly isolated $M M$ cells replacing MM cell lines, and similar results were observed (data not shown).

\section{Functional GJs enhance the colony formation of MM cells}

A clonogenic assay was also performed in order to evaluate the proliferation potential of the MM cells. The data showed that the BMSCs themselves promoted the survival and colony formation of MM cells. Interestingly, the number of colonies formed with MM-BMSCs was very much augmented by this cell support and was more than those with ND-BMSCs. The number of colonies formed in the ND- or MM-BMSCs feed layer was $78 \pm 7$ and $121 \pm 11$ respectively $(p<0.05)$. The number

B
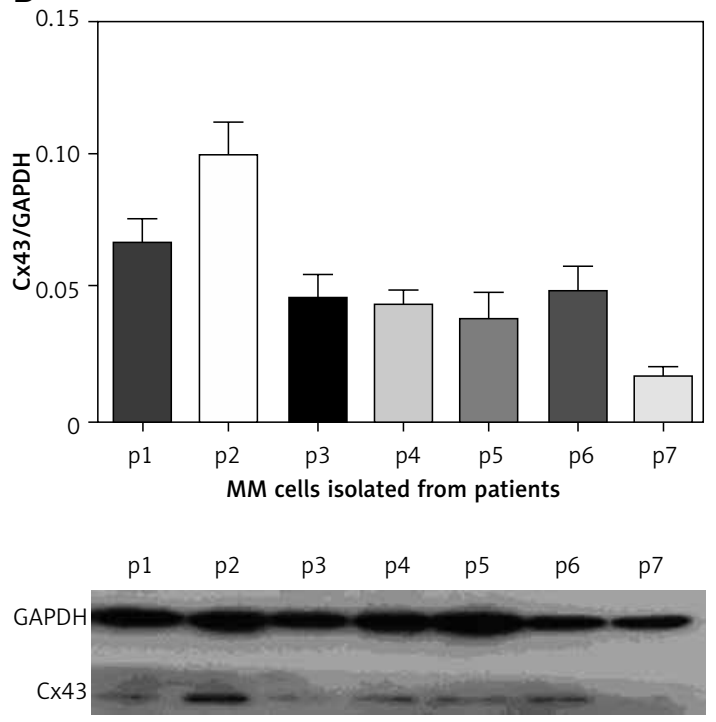

Figure 1. Cx43 expression on different MM cells and stromal cells. A - Real-time quantitative PCR assays showed that either MM cell lines or primary $M M$ cells expressed $\mathrm{C} \times 43$ at a low level besides RPMI 8226. BMSCs isolated from MM patients expressed higher levels of $\mathrm{C} \times 43$ than did BMSCs from normal donors. B - Western blot assay found that MM-BMSCs $(n=7)$ expressed a higher level of Cx43 than did BMSCs from normal donors $(n=5)$. C - All seven primary MM cells from patients expressed Cx43. Results are reported as relative quantitation of target $\mathrm{C} \times 43$ expression, normalized to an endogenous control (GAPDH) and relative to a calibrator 
of colonies decreased markedly to $47 \pm 5$ and 89 \pm 9 when the cells were incubated in the medium containing the GJ inhibitor heptanol (Figure 3).

\section{Blocking the GJ changes the cytokine secretion}

Cytometry bead array assays revealed that there were high levels of IL- 6 in supernatant of ND-BMSCs cultured alone, but the others were at low levels, and there was no IL-10 detected in the supernatant. The cytokines detected in the supernatant of RPMI 8226 cultured alone was also at a low level. The level of IL-6, IL-10 and SDF-1 $\alpha$ increased significantly after ND-BMSCs and MM cells were co-cultured for $24 \mathrm{~h}(p<0.05)$; in particular, the levels of IL-6 and IL-10 were higher as compared to those cells cultured alone $(p<0.001)$. There was no change of the level of bFGF and IL-17 before or after coculture. After blockage of the GJ with heptanol, the levels of IL- 6 and SDF- $1 \alpha$ returned to normal, but the level of IL-10 was higher than those cells cultured alone $(p<0.01)$ (Figure 4).

\section{BMSCs downregulate the sensitivity of $M M$ cells to bortezomib-induced apoptosis}

To investigate the effects of GJIC on BTZ-induced MM cell apoptosis, BTZ-induced cytotoxicity in the presence or absence of GJ inhibitor was examined. As shown in Figure 5, MM cells were

Figure 2-1
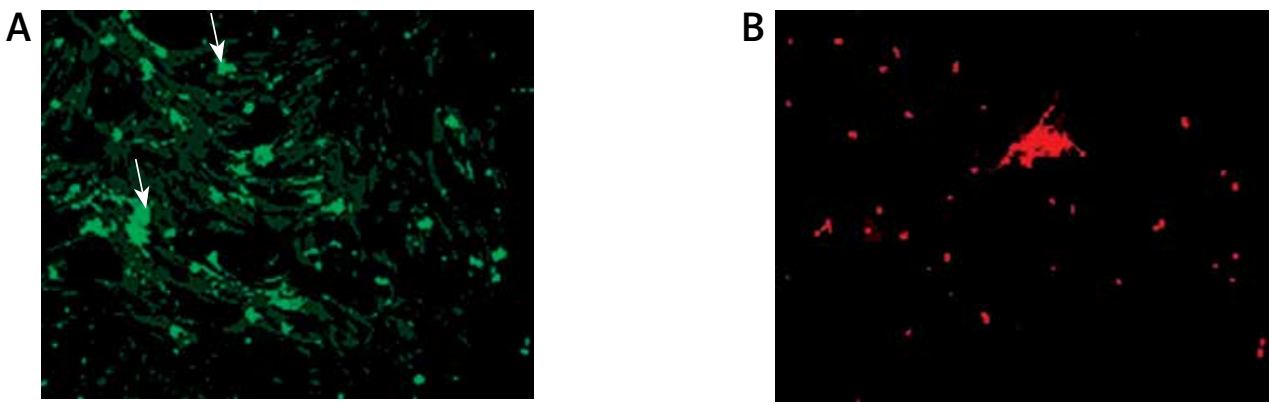

Figure 2-2
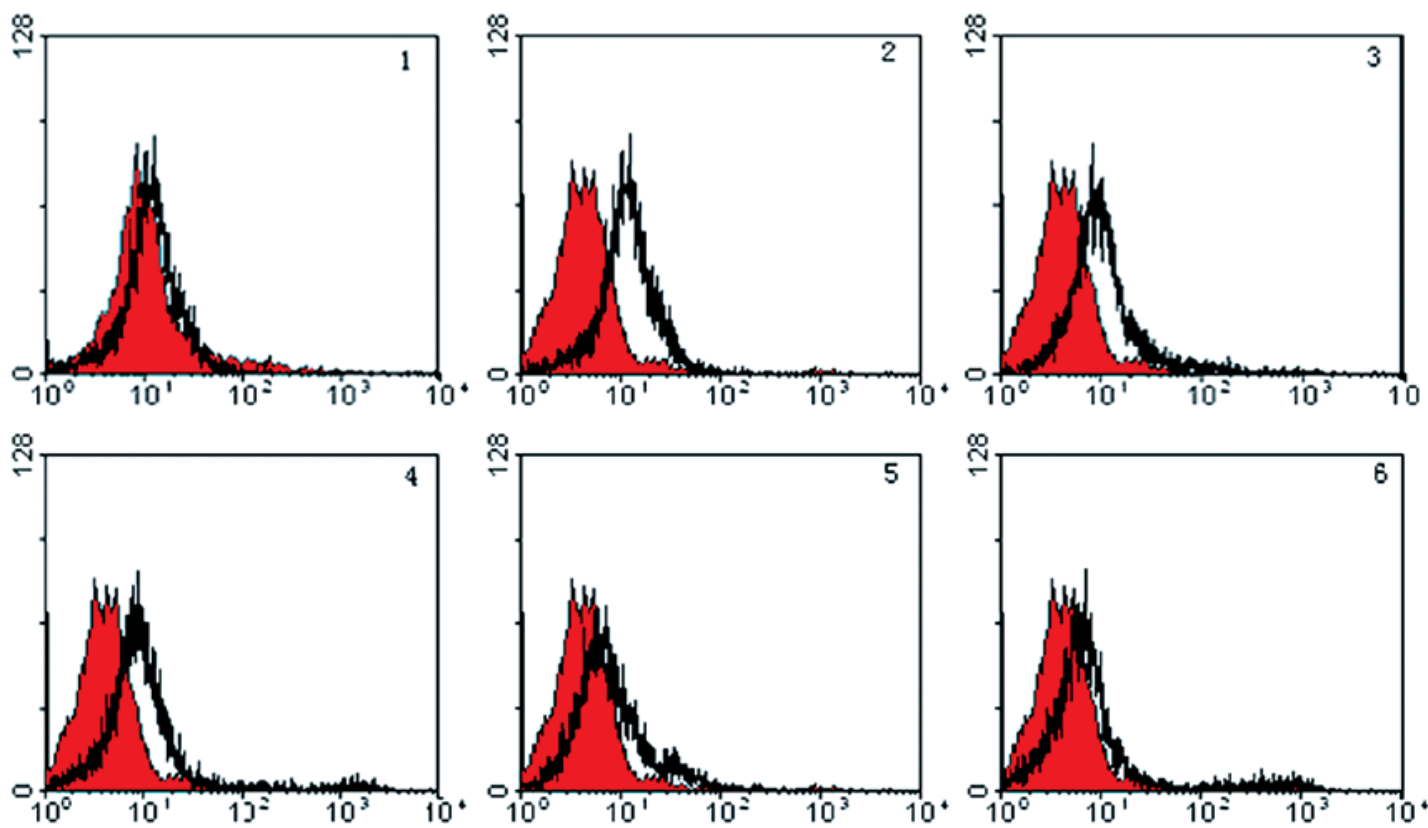

Figure 2. Gap junctions between MM and BMSCs. Microscopic photographs (2-1) from the double labeling of MM cells with calcein-AM (green) and Dil (red) show that dye transfer occurs from myeloma cells to BMSCs (A) and that dye is not permeating from the cells stained with Dil (B), confirming that GJIC is functional between the two cells, and that the dye-transfer was specific. FCM histograms (2-2) of RPMI 8266 after dual-labeled MM were administered onto BMSCs in a parachute assay, with GJs allowed to form. The FCM data show the percentage of BMSCS into which calcein-AM was transferred from MM cells after the cultures were treated with various concentrations of heptanol (0-100 mM/l). No. 1 displays the negative control samples. In the experimental cell population, many cells do not take up dye (overlapping with the negative control population), and shift to the right of the negative control population (which have taken up the dye (2-6)) 

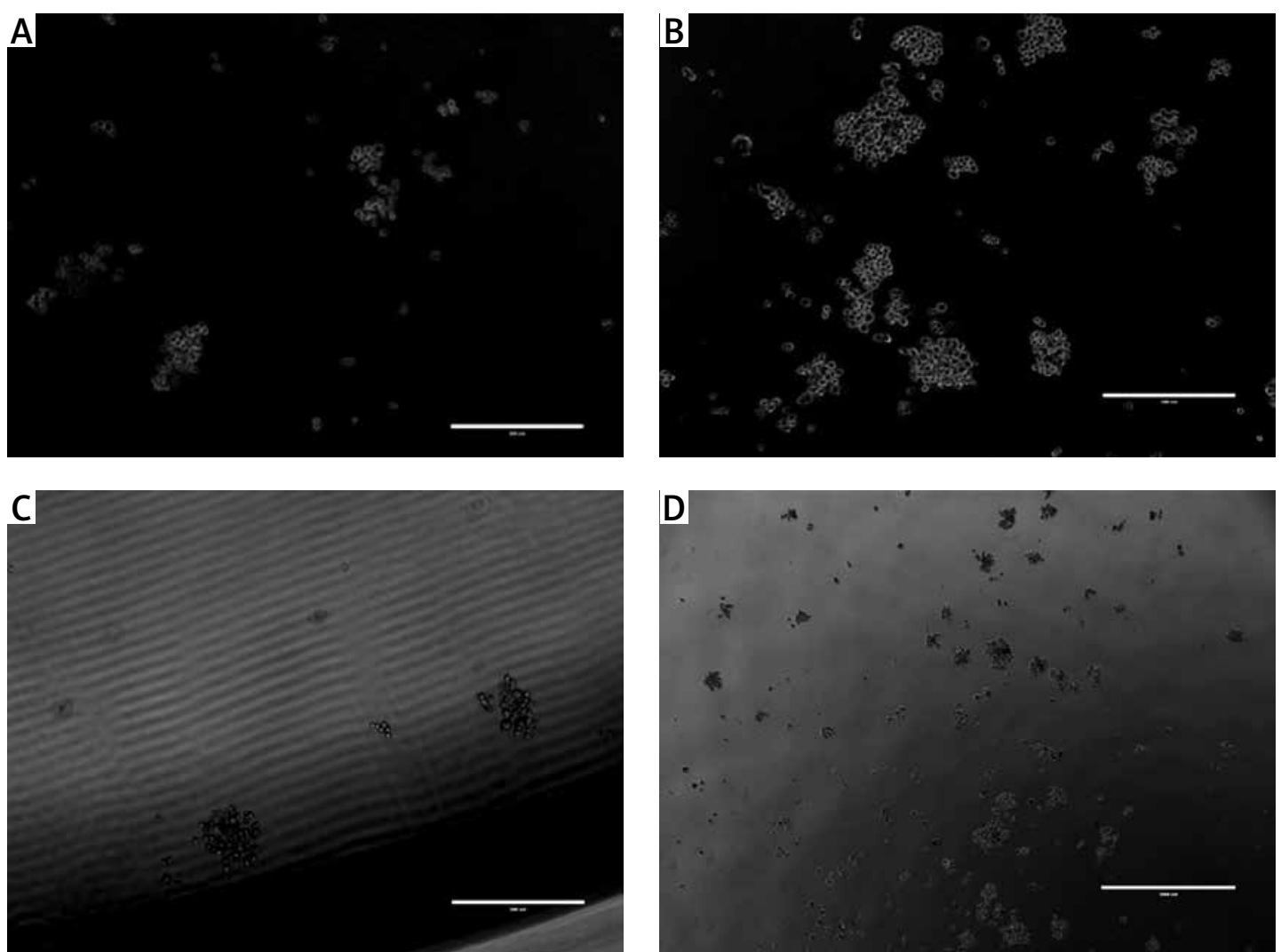

Figure 3. MM colony formation in vitro with or without GJ inhibitor. A clonogenic assay was also performed in order to evaluate the proliferation potential of the MM cells. Our data showed that MM cells generated more colonies on the BMSCs isolated from MM patients. A, C - Representative microscopic photographs show that colonies were formed by RPMI 8226 on MM-BMSCs (A) or ND-BMSCs (B) in the presence of GIIC inhibitor; B, D - MM cells generated more colonies when co-cultured with BMSCs in the absence of heptanol (B - MM-BMSCs and D - ND-BMSCS)

sensitive to BTZ-triggered apoptosis. After 24-h incubation of $M M$ cells in medium containing

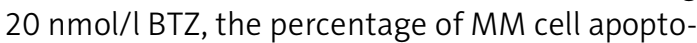
sis was approximately $66.9 \pm 0.86 \%$, significantly greater than the rate in controls $(6.5 \pm 0.2 \%$; $p<0.01)$. In the MM cell-BMSC co-culture model, BTZ caused a loss of cell viability significantly in the presence of BMSCs. The percentage of MM cell apoptosis in the presence of BMSCs was $19.9 \pm 0.7 \%$, markedly lower than the MM cell monoculture $(p<0.05)$, but higher than that of the controls. The BTZ-mediated cytotoxicity was partially recovered in the presence of GJ inhibitor, with a percentage of MM cell apoptosis of $31.6 \pm 0.5 \%$. Our results demonstrated that the MM-BMSCs were more effective than ND-BMSCs in protecting $M M$ cells from apoptosis. These experiments were also performed with MM cells cultured in medium with dexamethasone, and similar results were observed (data not shown). In further support of the role of GJs in promoting $M M$ cell survival, the GJ inhibitor heptanol also substantially reversed the loss of cell viability, an action which may contribute to MM chemoresistance.

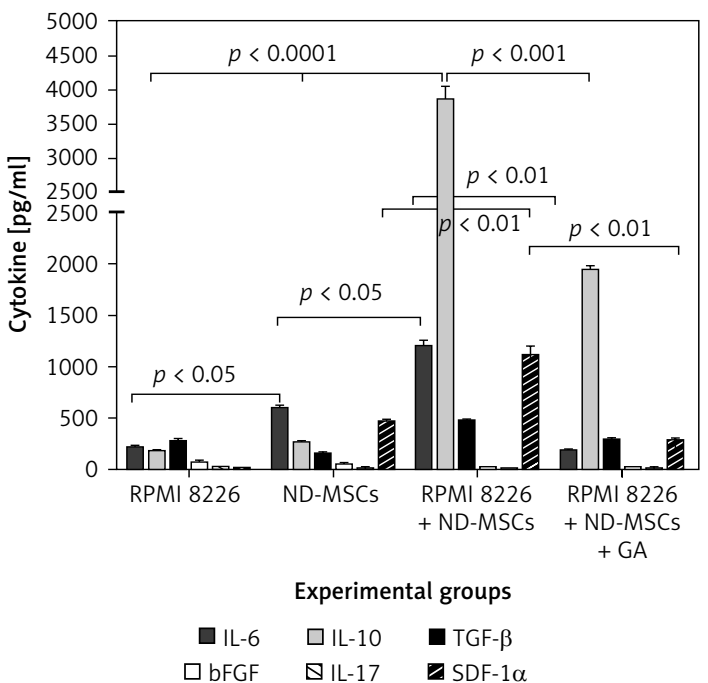

Figure 4. Changes of cytokine production in a coculture model with or without heptanol. We demonstrated that cytokine profiles of ND-BMSCs changed when they were incubated with MM cells and MM promoted the ND-BMSCs to secrete the cytokines which was in favor of the homing or growth of MM cells. The levels of IL-6, IL-10 and SDF-1 $\alpha$ increased significantly after ND-BMSCs and MM cells were co-cultured for $24 \mathrm{~h}(p<0.05)$ and decreased in the presence of the GJ inhibitor heptanol 
A

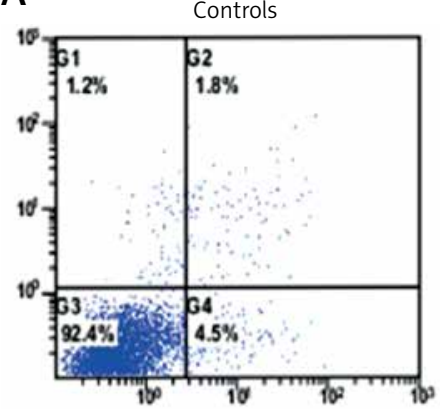

D

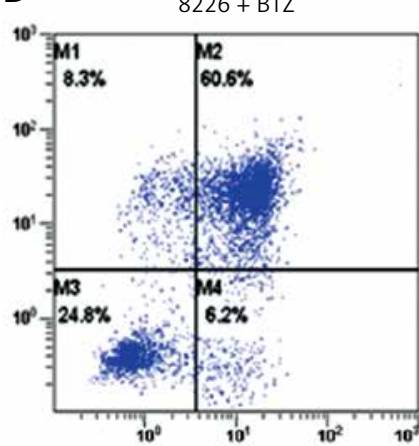

B

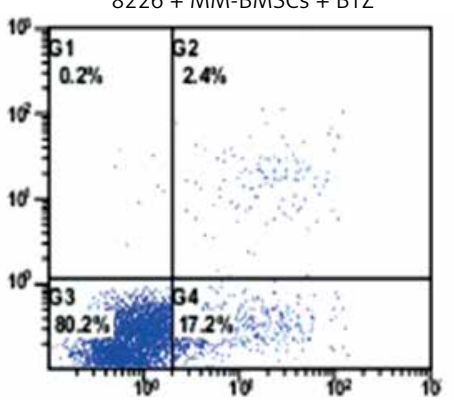

E $8226+$ MM-BMSCs + BTZ + heptanol

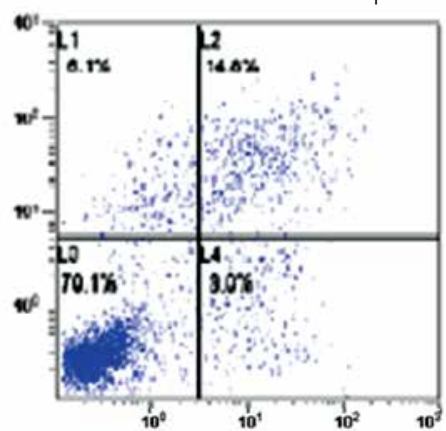

C

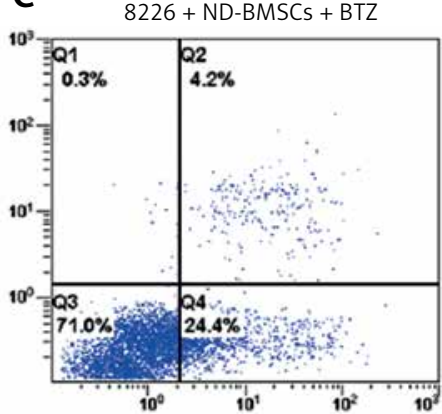

$\mathrm{F}$

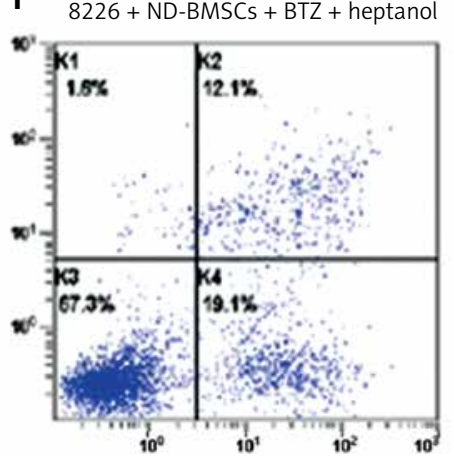

Figure 5. Effects of BMSCs on bortezomib (BTZ)-mediated MM cell apoptosis in vitro. RPMI 8266 cells were cultured in medium containing $20 \mathrm{nmol} / / \mathrm{BTZ}$ for $24 \mathrm{~h}$ and the apoptosis of MM cells was determined by FCM analysis, as shown in $5 \mathrm{E}$. The rate of apoptosis of MM cells cultured in the absence of BMSCs was approximately 76\% (D), but the ratio percentage of apoptosis decreased significantly when MM cells were co-cultured with BMSCs ( $p<0.05)$ (B and C), and increased when treated with $50 \mathrm{mM} / \mathrm{l}$ heptanol compared with the controls (4 and 6). A - Negative control

\section{Discussion}

Cellular membranes consist of GJs, intercellular channels assembled from the Cx family of transmembrane proteins, which allow the bidirectional passage of ions, metabolites, and secondary messengers in a process known as GIIC. The GJIC has been implicated in the regulation of homeostasis and a diverse array of cellular functions related to cell specialization, growth, and differentiation [24]. Cx43 is the predominant Cx expressed in BMSCs and OBs $[25,26]$, although other Cxs, such as Cx31 and Cx45, are also expressed in neonatal and adult BM stroma $[27,28]$. Altered expression of Cxs, such as reduced expression or aberrant localization, has been reported in many tumor tissues [29-32]. Such alterations impair cell-to-cell adhesion, which is indispensible for GJ formation. As a consequence, cellular clones selectively acquire the ability for rapid and unrestricted growth, along with disruption of tissue integrity. Recent evidence further suggests that Cxs may even facilitate latestage disease progression and metastasis [33, 34]. Our data indicate that Cx43 is expressed in MM cell lines and primary isolated MM samples. Interestingly, all BMSCs from MM patients expressed higher levels of $\mathrm{C} x 43$ than those from normal controls. This process may facilitate MM cells in forming GJs with Cx components distinct from the ones comprising GJs that involve only tumor cells. We confirmed the existence of GIC between MM cells and BMSCs with a dye transfer assay, which occurred in a dose-dependent manner. The GIC between the MM cells and BMSCs was specifically inhibited with heptanol. The mechanism by which this inhibition may affect cancer-related processes involving GJIC requires further study.

Cancer cells are a heterogeneous population by nature, although they share common malignant features, such as uncontrolled proliferation, poor differentiation, and potential invasiveness. Recent studies have revealed that MM stem cells exist in a BM niche, which gives rise to progenitor, committed, or differentiated MM cells and contributes to their heterogeneity. Although there are many factors reported to be involved in intercellular communication in eukaryotic cells $[35,36]$, our study revealed that $\mathrm{C} \times 43$ was expressed heterogeneously and aberrantly in MM cells. Cx43 mRNA expression was observed in RPMI 8266, XG7, and U266 cells. Concomitantly, approximately $85 \%$ of freshly isolated MM cells expressed Cx43 (i.e., 6 out of 7 patients with MM). Interestingly, Cx43 expression in BMSCs isolated from patients with $M M$ was greater than that observed in both $M M$ 
cells and normal BMSCs. The results of the current study indicate that the microenvironment created by BMSCs plays an important role in supporting the growth of myeloma stem cells. Although it has been reported that myeloma stromal cells contribute to mature myeloma cell growth and chemoresistance, little is known about the interplay between BMSCs and myeloma stem cells. Our findings demonstrated that BMSCs increased the colony-forming ability and percentages of myeloma stem cells recovered in vitro to a greater extent than control or heptanol-treated BMSCs.

The MM microenvironment is formed by clonal plasma cells, extracellular matrix proteins, including collagen, fibronectin and laminin, and BM stromal cells, which are intimately involved in all biological stages of intramedullary growth. Moreover, binding of plasma cells to BMSCs triggers transcription and secretion of cytokines by the latter and mediates plasma cell growth, survival, and drug resistance, as well as BM angiogenesis. Studies demonstrated abnormal production of IL- 6 that was thought to be dependent on close contact with tumor cells, supporting a paracrine role of this cytokine in tumor growth and survival $[37,38]$. The source of IL- 6 was considered to be stromal 'elements' without further characterization. Our data demonstrated that BMSCs indeed take part in the overproduction of IL-6. In addition to this, other cytokines such as IL-10 and SDF-1 $\alpha$ were abnormally secreted in a co-culture manner, and the level of those cytokines decreased in medium containing the GJ inhibitor heptanol. It is reported for the first time that the gap junction played an important role in production of cytokines. The findings of this study may be important in the therapeutic modalities of multiple myeloma.

The proteasome inhibitor BTZ is an effective first-line therapy for the treatment of MM [39, 40]. Despite BTZ's greater effectiveness than most other chemotherapeutic agents in the treatment of certain hematological malignancies, intrinsic and acquired resistance remain significant limitations to treatment [41, 42]. Additionally, the mechanisms behind proteasome inhibitor resistance remain poorly understood. Data from various resistant cell lines generated by multiple investigators have implicated the upregulation of proteasome subunits and/or mutation of the $\beta 5$-subunit-encoding gene PSMB5 in this process [43-45]. Our data indicated that no substantial impact was observed on proliferation of MM cells, whether or not the GIIC with BMSCS was blocked with heptanol. However, the percentage of MM cells was significantly reduced in cultures treated with heptanol compared with the controls, and the MM cells were sensitive to BTZ-triggered apoptosis. In a co-culture model, BTZ significantly reversed a loss of cell viability when co-cultured in presence of BMSCs, although the cytotoxicity was partially recovered in the presence of a GJ inhibitor. The latter result provides further support for the role of GJs in promoting MM cell survival, where the GJ inhibitor heptanol markedly reversed the loss of cell viability, a process which may contribute to MM chemoresistance. Therefore, GIIC blockage of MM cell communication with BMSCs induces MM cell sensitivity to BTZ. It is apparent that CXs, in particular $\mathrm{Cx} 43$, not only affect channel-independent MM cell migration, but also exert profound effects on drug resistance in malignant plasma cells. Thus, the microenvironment of the BM plays an integral role in the pathogenesis of $M M$, stimulating a vicious feedback cycle of cytokine production, tumor growth, and bone destruction. Based on our data and those of other groups [46-48], we have demonstrated that the tight interaction between MM cells and BMSCs, hematopoietic niche component, endothelial cells, and the surrounding extracellular matrix leads to so-called cell adhesion-mediated drug resistance. Therefore, the specific targeting of GIC in BMSCS may be effective in inhibiting the protective effects of BMSCs and may improve therapeutic outcomes in hematological malignancies.

\section{Acknowledgments}

The author wishes to thank Zhang $\mathrm{H}$ for his technical assistance.

This study was supported by grants of the $\mathrm{Na}$ tional Nature Science Foundation of China (NFSC, No. 81272631) and in part by Jiangsu Province Special Program of Medical Science (BL 2012005).

\section{Conflict of interest}

The author declares no conflict of interest.

\section{References}

1. Fonseca R, Bailey RJ, Ahmann GJ, et al. Genomic abnormalities in monoclonal gammopathy of undetermined significance. Blood 2002; 100: 1417-24.

2. Zhan F, Hardin J, Kordsmeier B, et al. Global gene expression profiling of multiple myeloma, monoclonal gammopathy of undetermined significance, and normal bone marrow plasma cells. Blood 2002; 99: 1745-57.

3. Keats JJ, Reiman T, Belch AR, Pilarski LM. Ten years and counting: so what do we know about $\mathrm{t}(4 ; 14)$ (p16;q32) multiple myeloma. Leuk Lymphoma 2006; 47: 2289-300.

4. Fowler JA, Edwards CM, Croucher PI. Tumor-host cell interactions in the bone disease of myeloma. Bone 2011; 48: 121-8

5. Fowler JA, Mundy GR, Lwin ST, Lynch CC, Edwards CM. A murine model of myeloma that allows genetic manipulation of the host microenvironment. Dis Model Mech 2009; 2: 604-11.

6. Corre J, Mahtouk K, Attal M, et al. Bone marrow mesenchymal stem cells are abnormal in multiple myeloma. Leukemia 2007; 21: 1079-88. 
7. Civitelli R. Cell-cell communication in the osteoblast osteocyte lineage. Arch Biochem Biophys 2008; 473: 188-92.

8. Cancelas JA, Koevoet WLM, de Koning AE, Mayen AEM Rombouts EJC, Ploemacher RE. Connexin-43 gap junctions are involved in multiconnexin-expressing stromal support of hemopoietic progenitors and stem cells. Blood 2000; 96: 498-505.

9. Presley CA, Lee AW, Kastl B, et al. Bone marrow connexin-43 expression is critical for hematopoietic regeneration after chemotherapy. Cell Commun Adhes 2005; 12: 307-17.

10. Laird DW. Life cycle of connexins in health and disease. Biochem J 2006; 394: 527-43.

11. Wong RCB, Pera MF, Pebay A. Role of gap junctions in embryonic and somatic stem cells. Stem Cell Rev 2008; 4: 283-92.

12. Krenacs T, Rosendaal M. Connexin43 gap junctions in normal, regenerating, and cultured mouse bone marrow and in human leukemias: their possible involvement in blood formation. Am J Pathol 1998; 152: 993-1004.

13. Ciovacco WA, Goldberg CG, Taylor AF, et al. The role of Gap junctions in megakaryocyte-mediated osteoblast proliferation and differentiation. Bone 2009; 44: 80-6.

14. Hecht M, Heider U, Kaiser M, von Metzler I, Sterz J, Sezer O. Osteoblasts promote immigration and invasion of myeloma cells through upregulation of matrix metalloproteinases, urokinase plasminogen activator, hepatocyte growth factor and activation of p38 MAPK. Br Jaematol 2007; 138: 446-58.

15. Fu J, Wang P, Zhang X, et al. Myeloma cells inhibit osteogenic differentiating of mesenchymal stem cells and kil osteoblasts via TRAIL induced apoptosis. Arch Med Sci 2010; 6: 496-504.

16. Zhang X, Sun Y, Wang Z, et al. Upregulation of Cx43 expression in bone marrow mesenchymal stem cells plays a crucial role in adhesion and migration of multiple myeloma cells. Leuk Lymphoma 2015; 56: 211-8.

17. Horowitz MC, Coleman DL, Flood PM, Kupper TS, Jilka RL. Parathyroid hormone and lipopolysaccharide in duce murine osteoblast-like cells to secrete a cytokine indistinguishable from granulocyte-macrophage colony-stimulating factor. J Clin Invest 1989; 83: 149-57.

18. Simmons DJ, Kent GN, Jilka RL, Scott DM, Fallon M, Cohn DV. Formation of bone by isolated, cultured osteoblasts in millipore diffusion chambers. Calcif Tissue Int 1982; 34: 291-4

19. Horowitz MC, Xi Y, Pflugh DL, et al. Pax5-deficient mice exhibit early onset osteopenia with increased osteoclast progenitors. J Immunol 2004; 173: 6583-91.

20. Gu ZJ, Costes V, Lu ZY, et al. Interleukin-10 is a growth factor for human myeloma cells by induction of an oncostatin M autocrine loop. Blood 1996; 88: 3972-86.

21. Ziambaras K, Lecanda F, Steinberg TH, Civitelli R. Cyclic stretch enhances gap junctional communication between osteoblastic cells. J Bone Miner Res 1998; 13 218-28.

22. Shi Y, Yan H, Frost P, Gera J, Lichtenstein A. Mammalian target of rapamycin inhibitors activate the AKT kinase in multiple myeloma cells by up-regulating the insulin-like growth factor receptor/insulin receptor substrate-1/ phosphatidylinositol 3-kinase cascade. Mol Cancer Ther 2005; 4: 1533-40.

23. Ilvesaro J, Vaananen K, Tuukkanen J. Bone-resorbing osteoclasts contain gap junction a connexin-43. J Bone Miner Res 2000; 15: 919-26.
24. Urashima M, Chen BP, Chen S, et al. The development of a model for the homing of multiple myeloma cells to human bone marrow. Blood 1997; 90: 754-65.

25. Herve JC, Bourmeyster N, Sarrouilhe D, Duffy HS. Gap junctional complexes: from partners to functions. Prog Biophys Mol Biol 2007; 94: 29-65.

26. Yang J, Darley RL, Hallett M, Howard Evans W. Low connexin channel-dependent intercellular communication in human adult hematopoietic progenitor/stem cells: probing mechanisms of autologous stem cell therapy. Cell Commun Adhes 2009; 16: 138-45.

27. Lecanda F, Warlow PM, Sheikh S, Furlan F, Steinberg TH, Civitelli R. Connexin43 deficiency causes delayed ossification, craniofacial abnormalities, and osteoblast dysfunction. J Cell Biol 2000; 151: 931-44.

28. Presley CA, Lee AW, Kastl B, et al. Bone marrow connexin-43 expression is critical for hematopoietic regeneration after chemotherapy. Cell Commun Adhes 2005; 12: 307-17

29. Cancelas JA, Koevoet WL, de Koning AE, Mayen AE, Rom bouts EJ, Ploemacher RE. Connexin-43 gap junctions are involved in multiconnexin-expressing stromal support of hemopoietic progenitors and stem cells. Blood 2000; 96: 498-505.

30. Lecanda F, Towler DA, Ziambaras K, et al. Gap junctional communication modulates gene expression in osteoblastic cells. Mol Biol Cell 1998; 9: 2249-58.

31. Kanczuga-Koda L, Sulkowski S, Lenczewski A, et al. Increased expression of connexins 26 and 43 in lymph node metastases of breast cancer. J Clin Pathol 2006; 59: 429-33.

32. Ezumi K, Yamamoto H, Murata K, et al. Aberrant expres sion of connexin 26 is associated with lung metastasis of colorectal cancer. Clin Cancer Res 2008; 14: 677-84.

33. Westhoff MA, Zhou S, Bachem MG, et al. Identification of a novel switch in the dominant forms of cell adhesion-mediated drug resistance in glioblastoma cells. Oncogene 2008; 27: 5169-81.

34. Mesnil M, Crespin S, Avanzo JL, et al. Defective gap junctional intercellular communication in the carcinogenic process. Biochim Biophys Acta 2005; 1719: 125-45

35. Belting $M$, Wittrup A. Nanotubes, exosomes, and nucleic acid-binding peptides provide novel mechanisms of intercellular communication in eukaryotic cells: im plications in health and disease. J Cell Biol 2008; 183: 1187-91.

36. Schara K, Jansa V, Sustar V, et al. Mechanisms for the formation of membranous nanostructures in cell-to-cell communication. Cell Mol Biol Lett 2009; 14: 636-56.

37. Lin JH, Takano T, Cotrina ML, et al. Connexin 43 enhanc es the adhesivity and mediates the invasion of malignant glioma cells. J Neurosci 2002; 22: 4302-11.

38. Richardson PG, Sonneveld P, Schuster MW, et al. Safety and efficacy of bortezomib in high-risk and elderly patients with relapsed multiple myeloma. $\mathrm{Br} J$ Haematol 2007; 137: 429-35.

39. Merchionne F, Perosa F, Dammacco F. New therapies in multiple myeloma. Clin Exp Med 2007; 7: 83-97.

40. Ruschak AM, Slassi M, Kay LE, Schimmer AD. Novel proteasome inhibitors to overcome bortezomib resistance. J Natl Cancer Inst 2011; 103: 1007-17.

41. Orlowski RZ, Kuhn DJ. Proteasome inhibitors in cancer therapy: lessons from the first decade. Clin Cancer Res 2008; 14: 1649-57.

42. Kale AJ, Moore BS. Molecular mechanisms of acquired proteasome inhibitor resistance J Med Chem 2012; 55 10317-27. 
43. Ri M, lida S, Nakashima T, et al. Bortezomib-resistant myeloma cell lines: a role for mutated PSMB5 in preventing the accumulation of unfolded proteins and fatal ER stress. Leukemia 2010; 24: 1506-12.

44. Perez-Galan P, Mora-Jensen H, Weniger MA, et al. Bortezomib resistance in mantle cell lymphoma is associated with plasmacytic differentiation. Blood 2011; 117 542-52.

45. Balsas P, Galan-Malo P, Marzo I, Naval J. Bortezomib resistance in a myeloma cell line is associated to PSMbeta5 overexpression and polyploidy. Leuk Res 2012; 36 212-8.

46. Zhang X, Liu Y, Si YJ, et al. Effect of Cx43 gene-modified leukemic bone marrow stromal cells on the regulation of Jurkat cell line in vitro. Leukemia Res 2012; 36: 198 204.

47. Spengeman JD, Green TD, McCubrey JA, Bertrand FE. ACtivated EGFR promotes the survival of B-lineage acute leukemia in the absence of stromal cells. Cell Cycle 2005; 4: 483-7.

48. Garrido SM, Appelbaum FR, Willman CL, Banker DE. Acute myeloid leukemia cells are protected from spontaneous and drug-induced apoptosis by direct contact with a human bone marrow stromal cell line (HS-5). Exp Hematol 2001; 29: 448-57. 